\title{
MORTALIDADE DE BLATTELLA GERMANICA (L.) (BLATTODEA: BLATTELLIDAE) EM FUNÇÃO DA PORCENTAGEM DE EXPOSIÇÃO AOS INSETICIDAS E DO TIPO DE SUPERFÍCIE, ANTES E APÓS A LAVAGEM
}

\author{
I.C. Silva, M.C. Ferreira, R.S. Parreira, N.M. Martinelli, W.C. Pazini
}

Universidade Estadual Paulista, Faculdade de Ciências Agrárias e Veterinárias, Departamento de Fitossanidade, Via de Acesso Prof. Paulo Donato Castellane, s/nº, CEP 14884-900, Jaboticabal, SP, Brasil. E-mail: mailto: wpazini@fcav.unesp.br

\section{RESUMO}

Entre as espécies sinantrópicas de baratas, a barata alemã, Blattella germanica (L., 1767), é a mais importante por desenvolver altas populações em habitações. Este trabalho objetivou avaliar a mortalidade de $B$. germanica exposta a diferentes áreas tratadas, com quantidade equivalente de inseticidas e verificar a mortalidade provocada por inseticidas em superfícies lisa e rugosa, antes e após lavagem. Os experimentos foram conduzidos no Departamento de Fitossanidade da UNESP/ FCAV de Jaboticabal, SP. No primeiro experimento, a aplicação dos inseticidas foi realizada sobre placas de Petri, com áreas de exposição ao inseticida de 25, 50, 75 e 100\%. No segundo, a aplicação foi realizada com pulverizador costal pressurizado, sobre superfícies lisa (de azulejo) e rugosa (de cimento), que posteriormente foram lavadas com detergente e água corrente. Foram utilizados os inseticidas cypermethrin (Cynoff 400 PM) 2,5 g p.c./L; deltamethrin (Deltagard WG 250) 1,0 g p.c./L; lambda-cyhalothrin (Icon 5 CE) 5,0 mL p.c./L e uma testemunha sem aplicação. Foram confinadas, por quinze minutos, cinco baratas adultas no primeiro experimento e dez no segundo. As avaliações de mortalidade foram realizadas $0,1,2,4,24,48$ e 72 horas após o confinamento. Assim, conclui-se que os inseticidas aplicados em 50\%, 75\% e 100\% das áreas resultaram em alta mortalidade de B. germanica. Os inseticidas cypermethrin, deltamethrin e lambda-cyhalothrin foram eficientes no controle de B. germanica, antes da lavagem, na superfície lisa. A eficiência de controle dos inseticidas foi maior na superfície lisa que na superfície rugosa. Após a lavagem, a eficiência diminuiu em ambas as superfícies.

PALAVRAS-CHAVE: barata alemã, manejo de pragas urbanas, tratamento domissanitário.

\section{ABSTRACT}

MORTALITY OF BLATTELLA GERMANICA (L.) (BLATTODEA: BLATTELLIDAE) IN RELATION TO THE PERCENTAGE OF EXPOSURE TO PESTICIDES AND TYPE OF SURFACE, BEFORE AND AFTER WASHING. Among the species of synanthropic cockroaches, the German cockroach, Blattella germanica (L., 1767) is the most important in terms of developing high populations in habitations. This study evaluated the mortality of $B$. germanica exposed to different areas treated with an equivalent amount of insecticides to verify the mortality caused by pesticides on smooth and rough surfaces, before and after washing. The experiments were carried out at the Departamento de Fitossanidade, UNESP/FCAV, Campus Jaboticabal, SP, Brazil. In the first experiment, the insecticide was applied to Petri dishes, with areas of exposure to the insecticide of 25, 50,75 and $100 \%$. The second application was performed by pressurized spraying on smooth and rough surfaces, which were then washed with detergent and water after treatment. The insecticides used were cypermethrin (Cynoff 400 AM) 2.5 c.p. g/L, deltamethrin (250 Deltagard WG) 1.0 c.p. g/L; lambda-cyhalothrin (Icon 5 EC) c.p. $5.0 \mathrm{~mL} / \mathrm{L}$ and an untreated plot (control). In the first experiment, 5 adult insects were enclosed for fifteen minutes, while 10 were enclosed in the second experiment. Mortality evaluations were performed at $0,1,2,4,24,48$ and 72 hours after confinement. It was found that the insecticides used in $50 \%, 75 \%$ and $100 \%$ of the area resulted in a high mortality of B. germanica. The insecticides cypermethrin, deltamethrin and lambda-cyhalothrin proved efficient for the control of B. germanica, before the washing of the smooth surface. The effectiveness of these insecticides was greater on a smooth surface than a rough one. After washing, the effectiveness was lower for both surfaces.

KEY WORDS: German cockroach, urban pest management, urban pest control. 


\section{INTRODUÇÃO}

As três espécies de baratas sinantrópicas mais importantes são a alemã Blattella germanica (L., 1767) (Blattodea: Blattellidae), a americana Periplaneta americana (L., 1758) (Blattodea: Blattidae) e a oriental Blatta orientalis (L., 1758) (Blattodea: Blattidae), essa última mais comum em regiões de clima temperado (MARICONI, 1999). Dentre estas, a barata alemã é a que vem causando maiores preocupações e prejuízos em países de clima subtropical e tropical como o Brasil, desenvolvendo altas populações em restaurantes, residências, hospitais e locais de armazenamento de alimentos (LOPES, 2005).

A barata alemã, $B$. germanica, é a espécie mais conhecida. Os adultos medem de 12 a $16 \mathrm{~mm}$ de comprimento e são de coloração castanho-amarelada, sendo machos e fêmeas alados. A fêmea carrega a ooteca presa ao abdômen até poucas horas antes da eclosão das ninfas (GuIMARÃES, 1984). Esta espécie pode reproduzir-se com maior rapidez do que qualquer outra espécie sinantrópica, característica que a torna a principal praga urbana em termos econômicos. Dependendo das condições ambientais, são necessários de 40 a 125 dias para completarem o ciclo. O adulto tem longevidade de até um ano e apresentam reprodução sexuada. Cada uma das ootecas pode conter de menos de 30 a mais de 40 indivíduos (GranovsKY, 1996).

As infestações de baratas podem ser controladas através da aplicação de produtos domissanitários, associado a modificações do ambiente, para privar os insetos de alimento e abrigo. Pequenas populações também podem ser controladas por iscas ou armadilhas (BEHBEHANI, 1997). Em condições sanitárias adequadas, a eficiência no tratamento de áreas infestadas é maior (SCHAL, 1988).

A higieneéfundamental para o controle da barata. Em edificações térreas é mais simples manter o controle do que em prédios de apartamentos, onde as baratas têm acesso a refúgios complexos. Entretanto, algumas medidas de limpeza, como a lavagem de pisos, podem reduzir a eficiência de inseticidas, requerendo conhecimento de seus efeitos.

O objetivo do presente trabalho foi avaliar a mortalidade de B. germanica exposta a diferentes áreas tratadas, com quantidade equivalente de inseticidas e verificar a mortalidade provocada por inseticidas em superfícies lisa e rugosa, antes e após lavagem.

\section{MATERIAL E MÉTODOS}

O experimento foi conduzido em laboratório do Departamento de Fitossanidade do Campus de Jaboticabal da UNESP/FCAV. A população de $B$. germanica utilizada foi criada a partir de setembro de
2006, proveniente de matrizes cedidas pelo Centro dePesquisa e Desenvolvimento deSanidade Vegetal do Instituto Biológico, São Paulo. Essa população é caracterizada como suscetível ao tratamento com inseticidas.

As baratas foram mantidas em caixas plásticas (56,1 L, Sanremo, ref. 975) untadas com vaselina nas bordas superiores internas para impedir o acesso à tampa, revestida com tecido de algodão branco para permitir a respiração dos insetos. Três embalagens para ovos (papelão) foram adaptadas para alojamento das baratas. A água foi disponibilizada através de dois bebedouros para pássaros, com chumaço de algodão na saída. O alimento, triturado em liquidificador (ração para gatos triturada, Maxi Cat, Alimento Premium), foi oferecido em uma placa de Petri de sete centímetros de diâmetro. Estes procedimentos, para criação de B. germanica em laboratório, foram adaptados dos relatados por Cornwell (1968).

Para a realização dos experimentos foram utilizados os inseticidas cypermethrin (Cynoff 400 PM) 2,5 g p.c./L; deltamethrin (Deltagard WG 250) 1,0 g p.c./L; lambda-cyhalothrin(Icon 5 CE) $5,0 \mathrm{~mL}$ p.c./L e uma testemunha sem aplicação. Os piretroides foram selecionados por serem os mais empregados no controle de baratas (GALLO et al., 2002).

A mortalidade de B. germanica, em função da área tratada foi verificada através da aplicação dos inseticidas, em torre de Potter sobre placas de Petri de $14 \mathrm{~cm}$ de diâmetro, com áreas de exposição ao inseticida de $25 \%, 50 \%, 75 \%$ e $100 \%$ e mais uma testemunha sem aplicação. $\mathrm{O}$ volume de aplicação foi de $0,5 \mathrm{~mL}$ por placa.

Para cada área de exposição foi mantida a mesma quantidade de produto comercial (área 100\% = concentração recomendada pelo fabricante, área $75 \%=$ concentração $\times 1,333$, área $50 \%=$ concentração $\times 2$, área $25 \%$ = concentração $\times 4$ ). As partes "não tratadas" das placas foram recobertas por um filme de polipropileno de espessura 0,05 mm, antes da aplicação.

Após a secagem das placas de Petri (período de uma hora), o filme plástico foi retirado e cinco indivíduos adultos (machos e fêmeas coletados aleatoriamente) foram confinados sobre a placa por um período de 15 minutos. Este procedimento assemelha-se ao adotado por SALMERON; ОмоTо (2004). Para fazer este procedimento, utilizou-se potes de plástico transparentes, furados na parte inferior e untado com vaselina líquida nos bordos, virado sobre a placa de Petri, com as baratas no interior. Após a exposição ao inseticida, as baratas foram anestesiadas $\mathrm{Com} \mathrm{CO}_{2}$ e transferidas para potes de plástico com tampas perfuradas, com suprimento de alimento e água. A mortalidade foi avaliada imediatamente após, 1, 2, 4, 24, 48 e 72 horas após a exposição. 
As mortalidades de B. germanica por inseticida, em superfície lisa (revestida de azulejo) e rugosa (piso cimento), antes e após lavagem, foram verificadas após a aplicação dos inseticidas com um pulverizador costal pressurizado $\left(\mathrm{CO}_{2}\right)$, equipado com um bico dejato plano contínuo 8002E (TPSpraying Systems Co.) sob uma pressão de $350 \mathrm{kPa}(50,8$ $\mathrm{lbf} / \mathrm{pol}^{2}$ ) e volume de aplicação de $40 \mathrm{~mL} / \mathrm{m}^{2}$. Este sistema de aplicação foi adaptado de POTENZA et al. (2003), que obtiveram eficiência residual de inseticidas no controle de B. germanica com a utilização da ponta de pulverização TXA8001 VK (Spraying Systems Co) numa pressão de $50 \mathrm{lbf} / \mathrm{pol}^{2}$ e volume de aplicação de $40 \mathrm{~mL} / \mathrm{m}^{2}$.

A aplicação dos inseticidas na superfície lisa (azulejo) foi realizada no lado externo da Unidade de Pesquisa e Ensino de Técnicas de Aplicação de Defensivos Agrícolas do Departamento de FitossanidadeFCAV/UNESP, sob temperatura média de $14,5^{\circ}$ C e UR $62 \%$. Cada piso possuía $0,137 \mathrm{~m}^{2}$ num total de 1,6 $\mathrm{m}^{2}$ para cada área pulverizada. A aplicação de inseticida na superfície rugosa (cimento) também foi realizada do lado externo da mesma Unidade, sob temperatura média de $31^{\circ} \mathrm{C}$ e UR $24 \%$. Cada área tratada continha $1,6 \mathrm{~m}^{2}$.

Aproximadamente uma hora após o tratamento (para a secagem das superfícies) foram confinados 10 indivíduos adultos (cinco machos e cinco fêmeas) em um pote de plástico transparente, furado na parte inferior e untado com vaselina líquida nos bordos. Depois de 15 minutos após a exposição ao inseticida, as baratas foram anestesiadas com $\mathrm{CO}_{2}$ e transferidas para potes de plástico com tampas perfuradas, sendo mantidas com suprimento de alimento e água. A mortalidade foi avaliada conforme descrito para a porcentagem de área tratada.

Após 24 horas da aplicação, as superfícies foram lavadas com água corrente e detergente neutro, com o auxilio de uma vassoura, simulando a lavagem normal de um piso em habitação. Depois de secas, 10 baratas adultas (cinco machos e cinco fêmeas) foram confinadas sobre as superfícies, procedendo-se a

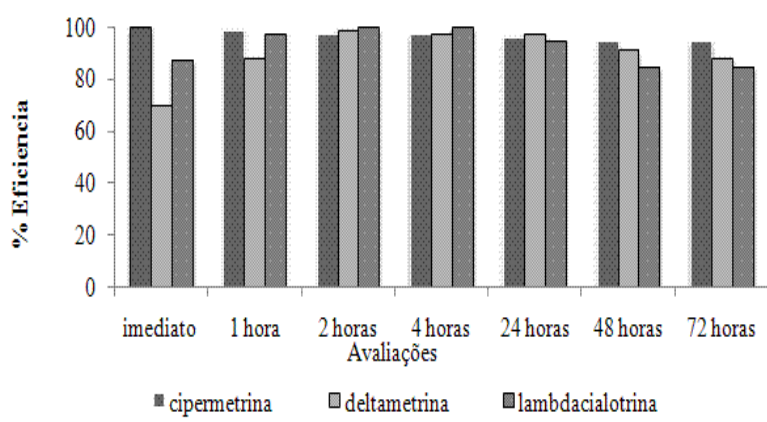

Fig. 1 - Porcentagem de eficiência dos inseticidas (média das áreas de exposição) nas avaliações da mortalidade de B. germanica. Jaboticabal, SP, 2007. transferência dos insetos para as avaliações conforme descrito anteriormente.

O delineamento experimental utilizado, em ambos os experimentos foi inteiramente casualizado com quatro repetições. Os resultados em porcentagem de mortalidade foram transformados em arco seno $\sqrt{\mathrm{p} / 100}$, submetidos à análise de variância e as médias comparadas pelo teste de Tukey $(p \leq 0,05)$. As percentagens de eficiência foram calculadas pela fórmula de Abbott (NAKANO et al., 1981).

\section{RESULTADOS E DISCUSSÕES}

$\mathrm{Na}$ avaliação imediatamente após a exposição, os inseticidas aplicados nas áreas de exposição 75\% e $100 \%$ apresentaram maiores mortalidades de $B$. germanica, mas não diferiram significativamente em relação à área de $50 \%$ (Tabela 1). Não houve diferença significativa entre as áreas de exposição $(25 \%, 50 \%$, $75 \%$ e $100 \%$ ), nas demais avaliações. Estes resultados diferem dos obtidos por PARREIRA et al. (2010) que avaliaram a mortalidade de B. germanica sob áreas de exposição em superfícies tratadas com inseticidas, nas dosagens recomendadas pelos fabricantes e verificaram que as mortalidades das baratas não diferiram significativamente entre as áreas de 100\% e $75 \%$, mas esta foi significativamente menor nas áreas de exposição $25 \%, 50 \%$, quando submetidas ao efeito residual de diversos inseticidas.

A maior eficiência, imediatamente após a exposição, foi obtida com o inseticida cypermethrin (100\%), enquanto que a menor eficiência imediata foi para deltamethrin (Fig. 1). Nas avaliações de 1 hora até as 72 horas de exposição de B. germanica, todos os inseticidas apresentaram eficiências superior a $80 \%$.

A porcentagem deeficiênciaobtidaatravés da média dosinseticidasapresentoumenores valoresondeforam tratados 25\% das áreas (Fig. 2). As áreas 50\% e 75\% tratadas apresentaram eficiências entre $80 \%$ a $100 \%$. A maior eficiência foi observada para a superfície 100\% tratada e a menor foi da superfície de $25 \%$.

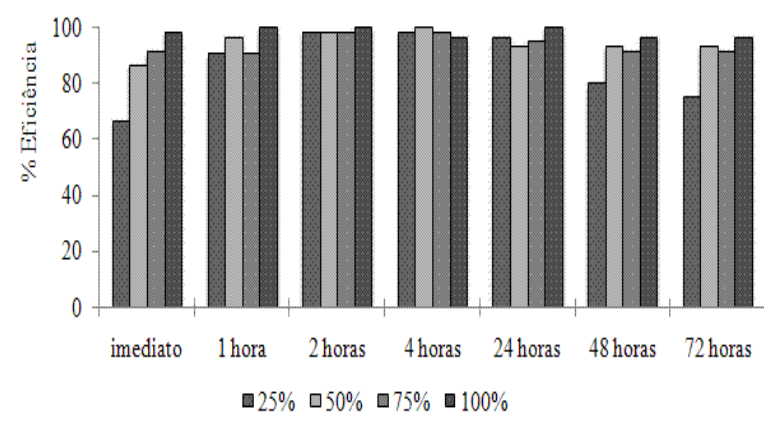

Fig. 2 - Porcentagem de eficiência (média dos inseticidas) sobre B. germanica em relação às áreas de exposição. Jaboticabal, SP, 2007. 


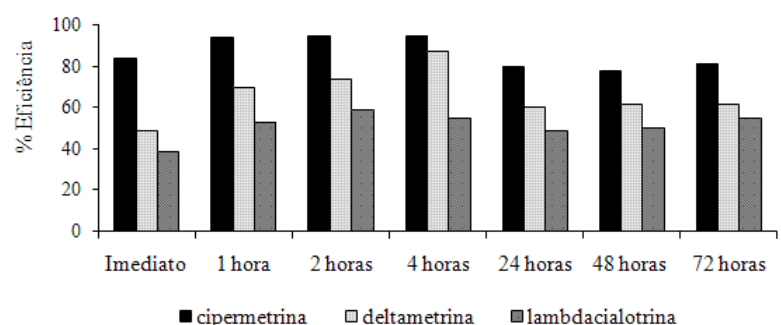

Fig. 3 - Porcentagem de eficiência dos inseticidas (média de sem e com lavagem) nas avaliações da mortalidade de B. germanica em superfície lisa. Jaboticabal, SP, 2007.

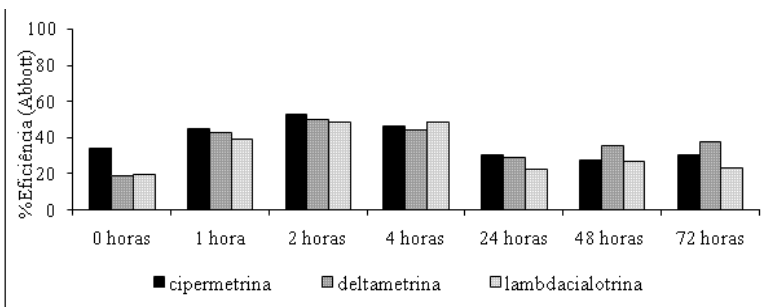

Fig. 5 - Porcentagem de eficiência dos inseticidas na mortalidade de Blattella germanica em superfície rugosa, sem e com lavagem. Jaboticabal, SP, 2007.

A lavagem da superfície lisa (azulejo) provocou uma redução significativa na eficiência dos inseticidas (Tabela 2). Os inseticidas lambda-cyhalothrin e deltamethrin resultaram em menor mortalidade de B. germanica na avaliação logo após a exposição em superfície lisa antes e após a lavagem. O inseticida cypermethrinapresentou umefeito "knock-down" que resultou em maiormortalidadedas baratas. Entretanto, o efeito "knock-down" de um produto nem sempre pode ser considerado como a melhor forma de avaliar a sua eficiência, porque este efeito pode não resultar efetivamente em mortalidade dos insetos (SALMERON; Омото, 2004). Para as avaliações dos horários de 24, 48 e 72 horas após a exposição, o inseticida cypermethrin apresentou maiores eficiências não diferindo significativamente do inseticida deltamethrin (Tabela 2).

A porcentagem de eficiência dos inseticidas (média de sem e com lavagem), na superfície lisa em todas as avaliações, foram maiores para o inseticida cypermethrin seguido por deltamethrin e lambdacyhalothrin (Fig. 3).

Com relação à lavagem da superfície lisa, a porcentagem de eficiência foi reduzida após lavagem (Fig. 4). Portanto, esta prática, que também é um item importante em estabelecimentos que trabalham com alimentos, diminuiu a eficiência dos inseticidas avaliados, requerendo ações alternativas para a manutenção do controle das baratas.

Assim, a aplicação deve ser priorizada no acessoe no abrigo destes insetos, além de manter estratégias de acondicionamento adequadas para alimentos e água que possam ser atraentes. Considerando que na maioria dos estabelecimentos de serviço de alimentação aberto ao público, como bares, restaurantes e

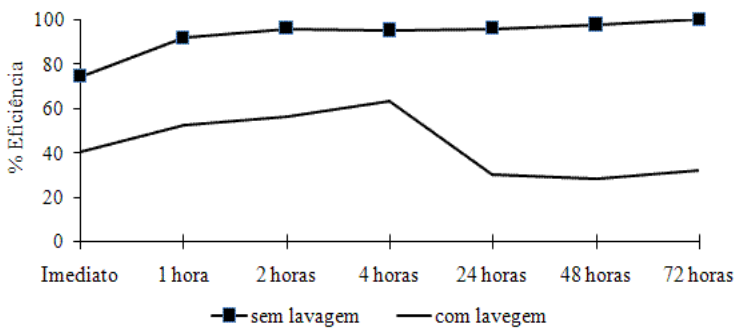

Fig. 4 - Porcentagem de eficiência (média dos inseticidas) na mortalidade de $B$. germanica em relação à superfície lisa, sem e com lavagem. Jaboticabal, SP, 2007.

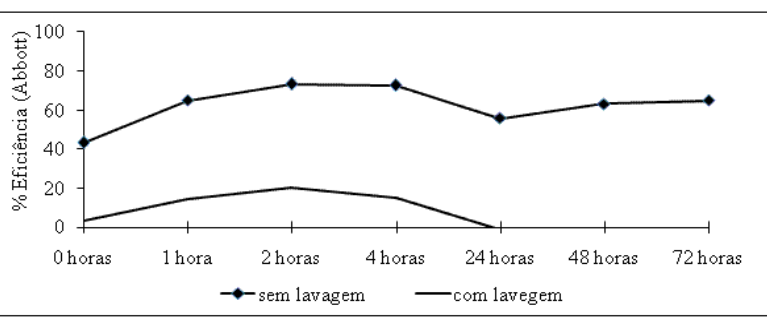

Fig. 6 - Porcentagens de eficiências (médias dos inseticidas) na mortalidade de Blattella germanica em relação à superfície de cimento, sem e com lavagem. Jaboticabal, SP, 2007.

padarias, a lavagem dos pisos acontece diariamente, pode-se recomendar que sejam determinadas estações temporais de tratamento domissanitário, com a finalidade de manter os inseticidas atuando por um período maior.

A superfície rugosa sem lavagem apresentou maior mortalidade de B. germanica diferindo significativamente da superfície rugosa com lavagem (Tabela 3). A mortalidade das baratas logo após a exposição com cypermethrin foi significativamente maior que os outros inseticidas. Nos demais horários, a mortalidade não diferiu entre os inseticidas, mas diferiram da testemunha.

As porcentagens de eficiência na superfície rugosa (média sem e com lavagem) de forma geral foram baixas para todos os horários de avaliação (Fig. 5).

As eficiências dos inseticidas diminuíram após a lavagem da superfície rugosa, em todas as avaliações (Fig. 6), assim como ocorreu na superfície lisa.

Os inseticidas aplicados na superfície lisa provocaram maior mortalidade de B. germanica diferindo significativamentequandoos mesmoinseticidasforam utilizados na superfície rugosa, sem a lavagem (Tabela 4). O inseticida cypermethrin resultou em maior mortalidade de B. germanica diferindo significativamente dos demais inseticidas, nas superfícies lisa e rugosa sem lavagem nas avaliações imediatamente, 2, 4 e 24 horas após a exposição (Tabela 4). Nas avaliações de 1 , 48 e 72 horas, o inseticida cypermethrin apresentou as maiores mortalidades, mas sem diferenças significativas ao inseticida deltamethrin. Também, o inseticida cypermethrin apresentou as maiores porcentagens de eficiência, acima de $80 \%$, em todas as avaliações nas superfícies lisa e rugosa antes da lavagem (Fig. 7). 


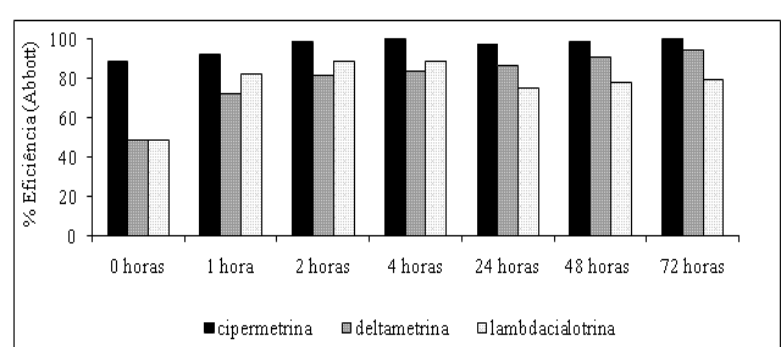

Fig. 7 - Porcentagem de eficiência dos inseticidas (média das superfícies lisa e rugosa) na mortalidade de Blattella germanica, sem lavagem. Jaboticabal, SP, 2007.

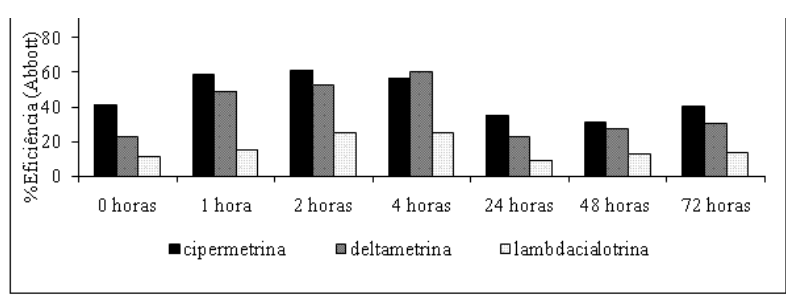

Fig. 9 - Porcentagem de eficiência dos inseticidas (média das superfícies lisa e rugosa) na mortalidade de Blattella germanica, com lavagem. Jaboticabal, SP, 2007.

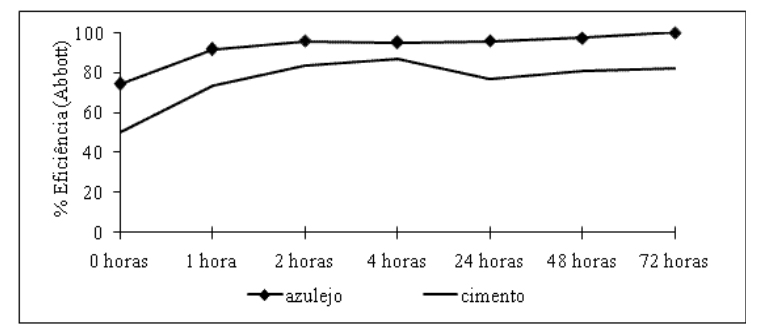

Fig. 8 - Porcentagem de eficiência dos inseticidas nas avaliações da mortalidade de Blattella germanica em relação às superfícies lisa erugosa, sem lavagem. Jaboticabal,SP, 2007.

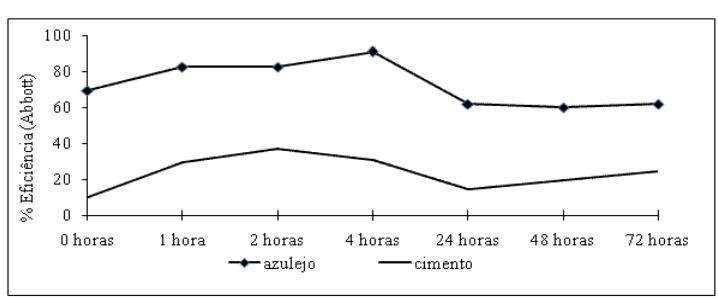

Fig. 10 - Porcentagem de eficiência dos inseticidas, nas avaliações da mortalidade de Blattella germanica em relação às superfícies lisa e rugosa, com lavagem. Jaboticabal, SP, 2007.

Tabela 1 - Porcentagem de mortalidade de Blattella germanica sob aplicação de inseticidas nas áreas de exposição. Jaboticabal, SP, 2007.

\begin{tabular}{lccccccc}
\hline \multirow{2}{*}{ Área de exposição } & \multicolumn{7}{c}{ Mortalidade após exposição 1,2 } \\
\cline { 2 - 8 } & imediato & 1 hora & 2 horas & 4 horas & 24 horas & 48 horas & 72 horas \\
\hline $25 \%$ & $45,79 \mathrm{~b}$ & $63,70 \mathrm{a}$ & $67,65 \mathrm{a}$ & $67,65 \mathrm{a}$ & $66,07 \mathrm{a}$ & $66,07 \mathrm{a}$ & $61,33 \mathrm{a}$ \\
$50 \%$ & $56,58 \mathrm{ab}$ & $63,70 \mathrm{a}$ & $65,28 \mathrm{a}$ & $62,46 \mathrm{a}$ & $61,33 \mathrm{a}$ & $62,18 \mathrm{a}$ & $62,18 \mathrm{a}$ \\
$75 \%$ & $59,75 \mathrm{a}$ & $63,70 \mathrm{a}$ & $67,65 \mathrm{a}$ & $67,65 \mathrm{a}$ & $62,90 \mathrm{a}$ & $59,75 \mathrm{a}$ & $59,74 \mathrm{a}$ \\
$100 \%$ & $65,28 \mathrm{a}$ & $66,86 \mathrm{a}$ & $66,86 \mathrm{a}$ & $64,49 \mathrm{a}$ & $66,86 \mathrm{a}$ & $63,70 \mathrm{a}$ & $63,70 \mathrm{a}$ \\
\hline Teste F área & $7,42^{* *}$ & $0,37 \mathrm{~ns}$ & $0,27 \mathrm{~ns}$ & $0,63 \mathrm{~ns}$ & $1,02 \mathrm{~ns}$ & $0,92 \mathrm{~ns}$ & $0,33 \mathrm{~ns}$ \\
DMS (5\%) & 11,34 & 9,72 & 8,15 & 12,07 & 9,75 & 10,46 & 10,87 \\
\hline Tratamentos & & & & & & & \\
\hline cypermethrin & $88,72 \mathrm{a}$ & $87,14 \mathrm{a}$ & $85,56 \mathrm{a}$ & $85,56 \mathrm{a}$ & $83,19 \mathrm{a}$ & $82,39 \mathrm{a}$ & $82,39 \mathrm{a}$ \\
deltamethrin & $61,26 \mathrm{c}$ & $79,23 \mathrm{a}$ & $87,14 \mathrm{a}$ & $81,95 \mathrm{a}$ & $86,35 \mathrm{a}$ & $86,35 \mathrm{a}$ & $81,60 \mathrm{a}$ \\
lambda-cyhalothrin & $76,14 \mathrm{~b}$ & $85,56 \mathrm{a}$ & $88,72 \mathrm{a}$ & $88,72 \mathrm{a}$ & $83,19 \mathrm{a}$ & $79,30 \mathrm{a}$ & $79,30 \mathrm{a}$ \\
Testemunha & $1,28 \mathrm{~d}$ & $6,02 \mathrm{~b}$ & $6,02 \mathrm{~b}$ & $6,02 \mathrm{~b}$ & $3,65 \mathrm{~b}$ & $3,65 \mathrm{~b}$ & $3,65 \mathrm{~b}$ \\
\hline Teste F produto & $165,30^{* *}$ & $229,67^{* *}$ & $351,38^{* *}$ & $153,82^{* *}$ & $243,59^{* *}$ & $203,35^{* *}$ & $179,93^{* *}$ \\
DMS (5\%) & 11,33 & 9,72 & 8,15 & 12,07 & 9,75 & 10,46 & 10,87 \\
\hline
\end{tabular}

${ }^{1}$ Médias seguidas pela mesma letra, na mesma coluna, não diferem entre si pelo teste de Tukey, ao nível de $5 \%$ de probabilidade.

${ }^{2}$ Dados transformados em arco seno $\sqrt{\mathrm{p} / 100}$.

Em relação às superfícies lisa e rugosa sem lavagem, para todos os horários de avaliação, as maiores eficiências foram apresentadas na superfície lisa (Fig. 8). Isto indicou que a superfície lisa apresentou uma maior disponibilidade de moléculas dos inseticidas.

As porcentagens de mortalidade de B. germanica sob a aplicação de inseticidas em superfícies lavadas foram significativamente maiores na superfície lisa que na superfície rugosa (Tabela 5). O inseticida cypermethrin apresentou, no geral, as maiores eficiências na mortalidade das baratas (superfícies lisa e rugosa, com lavagem), mas não diferiu significativamente do inseticida deltamethrin.

O inseticida cypermethrin apresentou, de forma geral, as maiores porcentagens de eficiências (médias das superfícies lisa e rugosa) após a lavagem das superfícies. (Fig. 9). 
Tabela 2 - Porcentagem de mortalidade de B. germanica sob a aplicação de inseticidas em superfície lisa sem e com lavagem. Jaboticabal, SP, 2007.

\begin{tabular}{|c|c|c|c|c|c|c|c|}
\hline \multirow{2}{*}{ Superfície lisa } & \multicolumn{7}{|c|}{ Mortalidade após exposição ${ }^{1,2}$} \\
\hline & Imediato & 1 hora & 2 horas & 4 horas & 24 horas & 48 horas & 72 horas \\
\hline sem lavagem & $46,18 \mathrm{a}$ & $58,66 \mathrm{a}$ & $62,16 \mathrm{a}$ & $60,47 \mathrm{a}$ & $61,56 \mathrm{a}$ & $63,76 \mathrm{a}$ & $67,05 \mathrm{a}$ \\
\hline com lavagem & $28,99 \mathrm{~b}$ & $34,26 \mathrm{~b}$ & $37,38 \mathrm{~b}$ & $41,11 \mathrm{~b}$ & $22,01 \mathrm{~b}$ & $21,35 \mathrm{~b}$ & $23,87 \mathrm{~b}$ \\
\hline Teste F & $19,34^{* *}$ & $31,27^{* *}$ & $36,70^{* *}$ & $33,76^{* *}$ & $94,05^{* *}$ & $140,60^{* *}$ & $101,28^{* *}$ \\
\hline DMS (5\%) & 8,06 & 9,01 & 8,44 & 6,89 & 8,42 & 7,38 & 8,86 \\
\hline \multicolumn{8}{|l|}{ Tratamentos } \\
\hline cypermethrin & $69,76 \mathrm{a}$ & $79,31 \mathrm{a}$ & $82,68 \mathrm{a}$ & $81,50 \mathrm{a}$ & $69,00 \mathrm{a}$ & $67,27 \mathrm{a}$ & $72,66 \mathrm{a}$ \\
\hline deltamethrin & $42,48 \mathrm{~b}$ & $58,79 \mathrm{~b}$ & $61,39 \mathrm{~b}$ & $72,06 \mathrm{a}$ & $54,44 \mathrm{ab}$ & $57,03 \mathrm{ab}$ & $57,03 \mathrm{ab}$ \\
\hline lambdacyalothrin & $37,20 \mathrm{~b}$ & $46,85 \mathrm{~b}$ & $54,09 \mathrm{~b}$ & $48,69 \mathrm{~b}$ & $42,81 \mathrm{~b}$ & $45,00 \mathrm{~b}$ & $51,23 \mathrm{~b}$ \\
\hline Testemunha & $0,90 \mathrm{c}$ & $0,90 \mathrm{c}$ & $0,90 \mathrm{c}$ & $0,90 \mathrm{c}$ & $0,90 \mathrm{c}$ & $0,90 \mathrm{c}$ & $0,90 \mathrm{c}$ \\
\hline Teste F & $52,49 * *$ & $57,88^{* *}$ & 72,20 ** & $116,73^{* *}$ & $51,55^{* *}$ & $66,72^{* *}$ & $52,39 * *$ \\
\hline DMS (5\%) & 15,24 & 17,02 & 15,95 & 12,99 & 15,91 & 13,95 & 16,73 \\
\hline
\end{tabular}

${ }^{1}$ Médias seguidas pela mesma letra, na mesma coluna, não diferem entre si pelo teste de Tukey, ao nível de $5 \%$ de probabilidade.

${ }^{2}$ Dados transformados em arco seno $\sqrt{\mathrm{p} / 100}$.

Tabela 3 - Porcentagem de mortalidade de B. germanica sob a aplicação de inseticidas em superfície rugosa sem e com lavagem. Jaboticabal, SP, 2007.

\begin{tabular}{|c|c|c|c|c|c|c|c|}
\hline \multirow{2}{*}{ Superfície rugosa } & \multicolumn{7}{|c|}{ Mortalidade $^{1,2}$} \\
\hline & Imediato & 1 hora & 2 horas & 4 horas & 24 horas & 48 horas & 72 horas \\
\hline sem lavagem & $35,11 \mathrm{a}$ & $45,61 \mathrm{a}$ & $52,08 \mathrm{a}$ & $54,99 \mathrm{a}$ & $48,88 \mathrm{a}$ & $53,25 \mathrm{a}$ & $55,87 \mathrm{a}$ \\
\hline com lavagem & $11,48 \mathrm{~b}$ & $22,52 \mathrm{~b}$ & $26,95 \mathrm{~b}$ & $24,28 \mathrm{~b}$ & $14,31 \mathrm{~b}$ & $16,82 \mathrm{~b}$ & $20,67 \mathrm{~b}$ \\
\hline Teste F & $46,60 * *$ & $27,93^{* *}$ & $48,38^{* *}$ & $75,14^{* *}$ & $93,22^{* *}$ & $72,98^{* *}$ & $62,04^{* *}$ \\
\hline DMS (5\%) & 7,14 & 9,03 & 7,44 & 7,31 & 7,39 & 8,80 & 9,23 \\
\hline \multicolumn{8}{|l|}{ Tratamentos } \\
\hline cypermethrin & $42,14 \mathrm{a}$ & $50,06 \mathrm{a}$ & $57,87 \mathrm{a}$ & $57,04 \mathrm{a}$ & $48,21 \mathrm{a}$ & $49,38 \mathrm{a}$ & $55,19 \mathrm{a}$ \\
\hline deltamethrin & $24,79 \mathrm{~b}$ & $45,35 \mathrm{a}$ & $51,08 \mathrm{a}$ & $48,41 \mathrm{a}$ & $42,33 \mathrm{a}$ & 47,99 a & $55,12 \mathrm{a}$ \\
\hline Lambda-cyhalothrin & $25,32 \mathrm{~b}$ & $39,94 \mathrm{a}$ & $48,06 \mathrm{a}$ & $52,20 \mathrm{a}$ & $34,96 \mathrm{a}$ & $39,67 \mathrm{a}$ & $38,65 \mathrm{a}$ \\
\hline testemunha & $0,90 \mathrm{c}$ & $0,90 \mathrm{c}$ & $0,90 \mathrm{c}$ & $0,90 \mathrm{c}$ & $0,90 \mathrm{c}$ & $3,09 \mathrm{~b}$ & $4,11 \mathrm{~b}$ \\
\hline Teste F & $24,01^{* *}$ & $26,49 * *$ & $52,26^{* *}$ & $54,13^{* *}$ & $34,94^{* *}$ & $25,93^{* *}$ & $28,99 * *$ \\
\hline DMS (5\%) & 13,49 & 17,04 & 14,05 & 13,81 & 13,97 & 16,64 & 17,43 \\
\hline
\end{tabular}

${ }^{1}$ Médias seguidas pela mesma letra, na mesma coluna, não diferem entre si pelo teste de Tukey, ao nível de $5 \%$ de probabilidade.

${ }^{2}$ Dados transformados em arco seno $\sqrt{\mathrm{p} / 100}$.

Tabela 4 - Porcentagem de mortalidade de Blattella germanica sob a aplicação de inseticidas em superfícies lisa e rugosa, sem lavagem. Jaboticabal, SP, 2007.

\begin{tabular}{|c|c|c|c|c|c|c|c|}
\hline \multirow{2}{*}{ Sem Lavagem } & \multicolumn{7}{|c|}{ Mortalidade $^{1,2}$} \\
\hline & Imediato & 1 hora & 2 horas & 4 horas & 24 horas & 48 horas & 72 horas \\
\hline Lisa & $46,18 \mathrm{a}$ & 58,66 a & $62,16 \mathrm{a}$ & $60,47 \mathrm{a}$ & $61,57 \mathrm{a}$ & $63,76 \mathrm{a}$ & $67,05 \mathrm{a}$ \\
\hline Rugosa & $35,10 \mathrm{~b}$ & $45,61 \mathrm{~b}$ & $52,00 \mathrm{~b}$ & $54,99 \mathrm{~b}$ & $48,89 \mathrm{~b}$ & $53,25 \mathrm{~b}$ & $55,87 \mathrm{~b}$ \\
\hline Teste F & $19,78^{* *}$ & 16,27 ** & $19,14^{* *}$ & $5,78^{*}$ & $23,43^{* *}$ & $14,83^{* *}$ & $16,46^{* *}$ \\
\hline DMS $(5 \%)$ & 5,14 & 6,68 & 4,79 & 4,7 & 5,41 & 5,63 & 5,69 \\
\hline \multicolumn{8}{|l|}{ Tratamentos } \\
\hline cipermethrin & $73,08 \mathrm{a}$ & $78,48 \mathrm{a}$ & $86,90 \mathrm{a}$ & 89,09 a & $84,71 \mathrm{a}$ & $86,90 \mathrm{a}$ & $89,09 \mathrm{a}$ \\
\hline deltamethrin & $44,33 \mathrm{~b}$ & $60,53 \mathrm{~b}$ & $66,26 \mathrm{~b}$ & $68,02 \mathrm{~b}$ & $72,59 \mathrm{~b}$ & $77,28 \mathrm{ab}$ & $81,50 \mathrm{ab}$ \\
\hline Lambda-cyhalothrin & $44,25 \mathrm{~b}$ & $68,64 \mathrm{ab}$ & $74,26 \mathrm{~b}$ & $72,89 \mathrm{~b}$ & $62,69 \mathrm{~b}$ & $66,74 \mathrm{~b}$ & $71,12 \mathrm{~b}$ \\
\hline testemunha & $0,90 \mathrm{c}$ & $0,90 \mathrm{c}$ & $0,90 \mathrm{c}$ & $0,90 \mathrm{c}$ & $0,90 \mathrm{c}$ & $3,09 \mathrm{c}$ & $4,11 \mathrm{c}$ \\
\hline Teste F & $142,69^{* *}$ & $116,52^{* *}$ & $274,04^{* *}$ & $291,46^{* *}$ & $202,94^{* *}$ & $192,27^{* *}$ & $199,67^{* *}$ \\
\hline DMS (5\%) & 9,72 & 12,62 & 9,05 & 8,89 & 10,22 & 10,64 & 10,75 \\
\hline
\end{tabular}


Tabela 5 - Porcentagem de mortalidade de Blattella germanica sob a aplicação de inseticidas em superfícies lisa e rugosa, com lavagem. Jaboticabal, SP, 2007.

\begin{tabular}{|c|c|c|c|c|c|c|c|}
\hline \multirow{2}{*}{ Com lavagem } & \multicolumn{7}{|c|}{ Mortalidade $^{1,2}$} \\
\hline & Imediato & 1 hora & 2 horas & 4 horas & 24 horas & 48 horas & 72 horas \\
\hline Lisa & $28.99 \mathrm{a}$ & $34,27 \mathrm{a}$ & $37,38 \mathrm{a}$ & $41,11 \mathrm{a}$ & $22,01 \mathrm{a}$ & $21,35 \mathrm{a}$ & $23,87 \mathrm{a}$ \\
\hline Rugosa & $11.48 \mathrm{~b}$ & $22,51 \mathrm{~b}$ & $26,95 \mathrm{~b}$ & $24,28 \mathrm{~b}$ & $14,31 \mathrm{a}$ & $16,82 \mathrm{a}$ & $20,67 \mathrm{a}$ \\
\hline Teste F & 14.59 ** & $4,99 *$ & $4,47^{*}$ & $15,35^{* *}$ & $2,63 \mathrm{~ns}$ & $0,87 \mathrm{~ns}$ & $0,34 \mathrm{~ns}$ \\
\hline DMS (5\%) & 9.47 & 10,86 & 10,19 & 8,87 & 9,81 & 10,01 & 11,46 \\
\hline \multicolumn{8}{|l|}{ Tratamentos } \\
\hline cipermethrin & $38,82 \mathrm{a}$ & $50,89 \mathrm{a}$ & $53,65 \mathrm{a}$ & $49,45 \mathrm{a}$ & $32,51 \mathrm{a}$ & $29,75 \mathrm{a}$ & $38,76 \mathrm{a}$ \\
\hline deltamethrin & $22.95 \mathrm{ab}$ & $43,62 \mathrm{a}$ & $46,21 \mathrm{ab}$ & $52,45 \mathrm{a}$ & 24,17 a & $27,74 \mathrm{a}$ & $30,65 \mathrm{a}$ \\
\hline Lambda-cyhalothrin & $18.27 \mathrm{bc}$ & $18,16 \mathrm{~b}$ & $27,89 \mathrm{~b}$ & $28,00 \mathrm{~b}$ & $15,07 \mathrm{ab}$ & $17,93 \mathrm{ab}$ & $18,76 \mathrm{ab}$ \\
\hline testemunha & $0,90 \mathrm{c}$ & $0,90 \mathrm{~b}$ & $0,90 \mathrm{c}$ & $0,90 \mathrm{c}$ & $0,90 \mathrm{~b}$ & $0,90 \mathrm{~b}$ & $0,90 \mathrm{~b}$ \\
\hline Teste F & 11.57 ** & $19,26^{* *}$ & $22,67 * *$ & $30,77^{* *}$ & $8,09 * *$ & $7,37^{* *}$ & $8,78^{* *}$ \\
\hline DMS (5\%) & 17.89 & 20,51 & 19,23 & 16,75 & 18,54 & 18,92 & 21,64 \\
\hline
\end{tabular}

${ }^{1}$ Médias seguidas pela mesma letra, na mesma coluna, não diferem entre si pelo teste de Tukey, ao nível de $5 \%$ de probabilidade.

${ }^{2}$ Dados transformados em arco seno $\sqrt{\mathrm{p} / 100}$.

Em relação às superfícies lisa e rugosa com lavagem, para todos os horários de avaliação, as maiores eficiências dos inseticidas foram apresentadas na superfície lisa (Fig. 10).

\section{CONCLUSÕES}

Os inseticidas aplicados em $50 \%, 75 \%$ e $100 \%$ das áreas resultam em alta mortalidade de B. germanica. Oinseticida cipermetrina apresenta efeito Knock down com eficiência. Os inseticidas cypermethrin, deltamethrin elambda-cyhalothrin são eficientes no controle B. germanica, antes da lavagem na superfície lisa.

A eficiência de controle dos inseticidas é maior na superfície lisa que na rugosa. Após a lavagem, a eficiência diminui nas superfícies lisa e rugosa.

\section{REFERÊNCIAS}

BEHBEHANI, K. Cockroaches: Unhygienic scavengers in human settlements. In: ROZENDAAL, J.A. (Ed.). Vector control-methods for use by individual and communities, Geneva: World Health Organization, 1997. p.288-301.

CORNWELL, P.B. The Cockroach: A laboratory insect and an industrial pest. London: The Rentokil Library, 1968. v.1,391p.

GALLO, D.; NAKANO, O.; SILVEIRA NETO, S.; CARVALHO, R.P.L.; BAPTISTA, G.C.; BERTI FILHO, E.; PARRA, J.R.P.; ZUCCHI, R.A.; ALVES, S.B.; VENDRAMIM, J.D.; MARCHINI, L.C.; LOPES, J.R.S.; OMOTO, C. Entomologia agrícola. Piracicaba: FEALQ, 2002. 920p.

GRANOVSKY, T.A. Controle de baratas em áreas urbanas. In: SIMPÓSIO LATINO-AMERICANO SOBRE CONTROLE DE PRAGAS URBANAS, 2., 1996, São Paulo. Anais. São Paulo, 1996. p.15-25.
GUIMARÃES, J.H. Baratas: manejo integrado em áreas urbanas. Agroquímica Ciba Geigy, v.25, p.20-24, 1984.

LOPES, R.B. Controle de Blattella germanica (L.) com Metarhizium anisopliae e inseticidas reguladores de crescimento. 2005. 121p. Tese (Doutorado em Agronomia) - Escola Superior de Agricultura "Luiz de Queiróz", Universidade de São Paulo, Piracicaba, 2005.

MARICONI, F.A.M. As baratas. In: MARICONI, F.A.M. (Ed.) Insetos invasores de residências. Piracicaba: FEALQ, 1999. v.6, p.13-33.

NAKANO, O.; SILVEIRA NETO, S.; ZUCCHI, R.A. Entomologia econômica. São Paulo: Ceres, 1981.314p.

PARREIRA, R.S.; FERREIRA, M.C.; MARTINELLI, N.M.; SILVA, I.C.; FERNANDES, A.P.; ROMANI, G.M. Mortalidade de Blattella germanica (L., 1767) (Blattodea: Blattellidae) sob diferentes áreas e períodos de exposição a diferentes inseticidas. Bioscience Journal, v.26, n.1, p.40-51, 2010.

POTENZA, M.R.; SANTOS, J.M.F.; SILVA, R.S.; ALVES, J.N . Avaliação de diferentes pontas de Pulverização na eficácia de inseticidas no tratamento de superfície para o controle de Blattella germanica (Dictyoptera: Blattellidae). Arquivos do Instituto Biológico, São Paulo, v.70, n.3, p.355-358, 2003.

SALMERON, E.; OMOTO, C. Mistura de deltametrina e clorpirifós no manejo da resistência de Blattella germanica (Linnaeus, 1757) (Dictyoptera: Blattellidae) a deltametrina. Entomotropica, v.19, n.2, p.85-89, 2004.

SCHAL, C. Relation among efficacy of insecticides, resistance levels and sanitation in the control of the german cockroach (Dictyoptera: Blattellidae). Journal of Economic Entomology, v.81, n.2, p.536-544, 1988. 